\title{
Intramolecular Cycloadditions of Cyclobutadiene with Dienes
}

\author{
John Limanto, ${ }^{\star}$ Kelli S. Khuong, ${ }^{\vee}$ K. N. Houk ${ }^{* \nabla}$ and Marc L. Snapper*»
}

Contribution from the Department of Chemistry, Merkert Chemistry Center, Boston College, 2609 Beacon Street, Chestnut Hill, MA 02467

and the Department of Chemistry and Biochemistry, The University of California, Los Angeles, California 90095-1569

\section{Supporting Information}

General Methods. All reactions were carried out under $\mathrm{N}_{2}$ atmosphere in oven-dried glassware $\left(120^{\circ} \mathrm{C}\right.$, $12 \mathrm{~h})$ using standard air-free manipulation techniques.

All reaction solvents were purchased from commercial sources and purified as follows: pentanes and $\mathrm{CH}_{2} \mathrm{Cl}_{2}$ were distilled over $\mathrm{CaH}_{2}$ under $\mathrm{N}_{2} ; \mathrm{THF}, \mathrm{Et}_{2} \mathrm{O}$, and benzene were distilled over $\mathrm{Na} / \mathrm{K}$ and benzophenone under $\mathrm{N}_{2}$ immediately prior to use. HPLC-grade acetone and $\mathrm{MeOH}$ were used in the cycloaddition without further purification and kept under $\mathrm{N}_{2}$ atmosphere. Chromatographic solvents, such as hexanes, pentanes, and $\mathrm{Et}_{2} \mathrm{O}$ were purified by simple distillation. All other commercially available reagents were purified by either simple distillation or recrystallization.

Concentration in vacuo refers to removal of solvent(s) using a Büchi rotary evaporator at 40-80 torr followed by the use of vacuum pump at approximately 1 torr. The use of brine refers to saturated (sat'd) aqueous (aq) $\mathrm{NaCl}$. Silica gel flash column chromatography was performed using EM Science Silica Gel 60 Geduran (35$75 \square \mathrm{m})$. Alumina gel flash chromatography was carried out using either Aldrich or Macherey-Nagel $58 \AA$ neutral, activated Brockmann type $\mathrm{I} \mathrm{Al}_{2} \mathrm{O}_{3}(\sim 150$ mesh). Analytical thin layer chromatography (TLC) was performed with $0.25 \mathrm{~mm}$ EM silica gel $60 \mathrm{~F}_{254}$ plates. Gas chromatography (GC) analyses were carried out using HP 5890 Series II spectrometer with a 30-m HP1 capillary fused column.

Proton $\left({ }^{1} \mathrm{H}\right)$ nuclear magnetic resonance (NMR) spectra were measured on either Varian Gemini-300 (300 MHz), Gemini-400 (400 MHz), or Gemini-500 (500 $\mathrm{MHz})$ instrument. Carbon $\left({ }^{13} \mathrm{C}\right)$ nuclear magnetic resonance (NMR) spectra were recorded using either Varian Gemini-75 (75 MHz), Gemini-100 (100 MHz), or Gemini-500 (500 MHz) instrument with complete proton decoupling mode. Chemical shifts are reported in ppm downfield from tetramethylsilane. Infrared (IR) spectra are reported in wavenumbers and measured on either a Nicolet 510 FT-IR or Perkin Elmer 1310 instrument. Gas chromatography-Mass spectroscopy (GC-MS) spectra were recorded using an HP 5890 Series II GC instrument coupled to an HP 5972 Mass Selective Detector. Melting points are reported without correction/calibration. Elemental analyses (Anal.) were performed by the analytical laboratory of Robertson Microlit Laboratoriess, Inc., Madison, NJ 07940.

Cycloaddition methods. Unless otherwise noted, all of the oxygen- and enone-containing tricarbonylcyclobutadiene-iron complex substrates were prepared according to the published procedures. ${ }^{1}$ The intramolecular cycloadditions were promoted by oxidative removal of the iron from its corresponding cyclobutadiene ligand according to one of the following methods:

Method A: CAN-promoted cycloadditions:

To a solution of the tricarbonylcyclobutadiene iron complex (1.0 equiv) under $\mathrm{N}_{2}$ atm in either HPLC-grade acetone or $\mathrm{MeOH}(1-3 \mathrm{mM})$ was added $\mathrm{CAN}$ (5.0 equiv) as a solid over $3 \mathrm{~min}$ at RT. The resulting orange solution was stirred for $10 \mathrm{~min}$, then neutralized with sat'd $\mathrm{NaHCO}_{3}$ solution and transferred to a separatory funnel containing $\mathrm{Et}_{2} \mathrm{O}$ and $\mathrm{H}_{2} \mathrm{O}$. The aqueous layer was separated and backextracted with $\mathrm{Et}_{2} \mathrm{O}(2 \mathrm{x})$. The combined organic layer was washed with $\mathrm{H}_{2} \mathrm{O}$, brine, dried over $\mathrm{MgSO}_{4} / \mathrm{K}_{2} \mathrm{CO}_{3}$, filtered and concentrated in vacuo, and purified by silica gel flash column chromatography.

Method B: TMAO-promoted cycloadditions:

To a solution of the tricarbonylcyclobutadiene iron complex (1.0 equiv) under $\mathrm{N}_{2}$ atm in HPLC-grade acetone (1-40 mM) was added a portion of TMAO (4-10 equiv). The resulting reaction mixture was refluxed for $6 \mathrm{~h}$, treated with a second portion of TMAO (4-10 equiv), and refluxed for additional 6-18 h. Once all starting material has been consumed as judged by TLC and GC, the resulting brown suspension was cooled to RT and then transferred to a separatory funnel containing $\mathrm{Et}_{2} \mathrm{O}$ and sat'd $\mathrm{NaHCO}_{3}$ solution. The aqueous layer was separated and backextracted with $\mathrm{Et}_{2} \mathrm{O}$. The combined organic layer was washed with $\mathrm{H}_{2} \mathrm{O}$, brine, dried over $\mathrm{MgSO}_{4} / \mathrm{K}_{2} \mathrm{CO}_{3}$, filtered, concentrated in vacuo, and purified by silica gel flash column chromatography.

Thermal rearrangements. In the cases of cyclobutadiene-diene cycloadditions, where two mode of cyclizations are operative, thermal isomerization of the $[2+2]$ products formed during the cycloadditions to the corresponding identical [4+2] cycloadduct were carried 
out by heating the [2+2] substrates to $130{ }^{\circ} \mathrm{C}$ in pentane in a sealed tube for 2-3 hours.

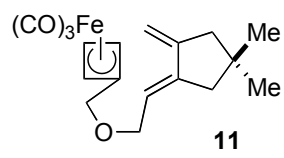

$11{ }^{1} \mathbf{H}$ NMR (400 MHz, $\left.\mathrm{CDCl}_{3}\right): \square 5.95$ $(1 \mathrm{H}, \mathrm{tt}, J=6.8,2.6 \mathrm{~Hz}), 5.34(1 \mathrm{H}$, br m), $4.88(1 \mathrm{H}$, br m), $4.18(2 \mathrm{H}, \mathrm{s}), 4.08(1 \mathrm{H}, \mathrm{s}), 4.07(2 \mathrm{H}, \mathrm{d}, J=6.8 \mathrm{~Hz}), 3.77$ $(2 \mathrm{H}, \mathrm{s}), 2.23(1 \mathrm{H}, \mathrm{m}), 2.20\left(1 \mathrm{H}\right.$, br s), $1.00(6 \mathrm{H}, \mathrm{s}) \cdot{ }^{13} \mathrm{C}$ NMR (100 MHz, $\mathrm{CDCl}_{3}$ ): $\square$ 214.4, 128.3, 128.0, 116.4, 104.3, 72.4, 68.7, 65.8, 65.1, 62.7, 48.6, 45.1, 36.6, 28.0. IR ( $\mathrm{NaCl}$, thin film): 2954, 2865, 2046, 1965, 1486, 1364 , 1087, 614, $589 \mathrm{~cm}^{-1}$. Anal. Calcd. for $\mathrm{C}_{18} \mathrm{H}_{20} \mathrm{O}_{4} \mathrm{Fe}: \mathrm{C}$, 60.67; H, 5.62. Found: C, 60.41; H, 5.83.

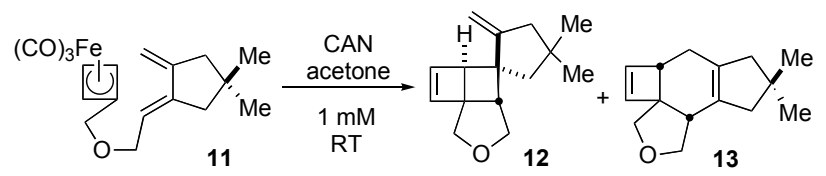

A solution of diene ether $11(30 \mathrm{mg}, 0.07 \mathrm{mmol}, 1.0$ equiv) and CAN (190 mg, $0.35 \mathrm{mmol}, 5.0$ equiv) in acetone $(70 \mathrm{~mL}, 1 \mathrm{mM})$ was treated according to the general cycloaddition procedure (method A) and purified by $\mathrm{SiO}_{2}$ gel gradient flash column chromatography (40:1; 20:1 pentanes: $\left.\mathrm{Et}_{2} \mathrm{O}\right)$ to give the two cycloadducts $(\mathbf{1 2 : 1 3}=$ $2.8: 1.0,15 \mathrm{mg}$ combined, $86 \%$ ) as colorless oils. The $[2+2]$ cycloadduct was isomerized to the corresponding identical [4+2] cycloadduct according to the general thermal rearrangement procedure ( $90 \%$ yield).

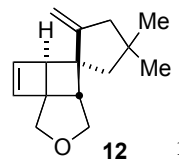

$J=2.4,1.5 \mathrm{~Hz}), 6.22(1 \mathrm{H}, \mathrm{d}, J=2.4 \mathrm{~Hz}), 4.83(1 \mathrm{H}, \mathrm{br})$ $4.78(1 \mathrm{H}, \mathrm{dm}, J=1.0 \mathrm{~Hz}), 4.13(1 \mathrm{H}, \mathrm{d}, J=10.3 \mathrm{~Hz}), 3.81$ $(1 \mathrm{H}, \mathrm{d}, J=9.9 \mathrm{~Hz}), 3.61(1 \mathrm{H}, \mathrm{dd}, J=10.3,6.6 \mathrm{~Hz}), 3.58$ $(1 \mathrm{H}, \mathrm{d}, J=9.9 \mathrm{~Hz}), 2.78(1 \mathrm{H}$, br s), $2.56(1 \mathrm{H}, \mathrm{d}, J=6.6$ $\mathrm{Hz}), 2.23(1 \mathrm{H}, \mathrm{ddd}, J=15.7,3.5,2.1 \mathrm{~Hz}), 2.13(1 \mathrm{H}, \mathrm{dm}$, $J=15.7 \mathrm{~Hz}), 1.82(1 \mathrm{H}, \mathrm{d}, J=12.8 \mathrm{~Hz}), 1.61(1 \mathrm{H}, \mathrm{d}, J=$ $12.8 \mathrm{~Hz}), 1.02(3 \mathrm{H}, \mathrm{s}), 0.98(3 \mathrm{H}, \mathrm{s}) .{ }^{13} \mathrm{C}$ NMR $(75 \mathrm{MHz}$, $\left.\mathrm{CDCl}_{3}\right): \square 157.8,138.4,136.7,106.8,70.6,69.7,60.0$, 57.7, 51.5, 49.6, 49.3, 45.1, 37.2, 29.3, 28.8. IR $(\mathrm{NaCl}$, thin film): 3090, 3023, 2951, 2842, 1650, 1465, 1371, 1087, 1025, $757 \mathrm{~cm}^{-1}$. GC-MS $(70 \mathrm{eV}) \mathrm{mlz}(\%$ rel intensity): $216\left(\mathrm{M}^{+}, 9\right), 201$ (40), 187 (19), 171 (35), 157 (27), 143 (34), 135 (40), 128 (46), 119 (60), 105 (50), 91 (93), 77 (92), 65 (40), 53 (43), 39 (100). Anal. Calcd. for $\mathrm{C}_{15} \mathrm{H}_{20} \mathrm{O}: \mathrm{C}, 83.23$; H, 9.26. Found: C, 82.86; H, 9.32.

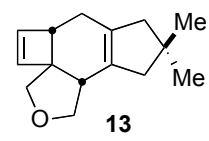

${ }^{1} \mathbf{H}$ NMR (400 MHz, $\left.\mathrm{CDCl}_{3}\right): \square 6.03(1 \mathrm{H}$, $\mathrm{dd}, J=2.9,1.3 \mathrm{~Hz}), 5.84(1 \mathrm{H}, \mathrm{d}, J=2.9 \mathrm{~Hz}), 4.01(1 \mathrm{H}, \mathrm{t}$,
$J=7.4 \mathrm{~Hz}), 3.88(1 \mathrm{H}, \mathrm{d}, J=7.3 \mathrm{~Hz}), 3.82(1 \mathrm{H}, \mathrm{d}, J=7.5$ $\mathrm{Hz}), 3.51(1 \mathrm{H}, \mathrm{dd}, J=12.4,7.1 \mathrm{~Hz}), 3.05(1 \mathrm{H}, \mathrm{d}, J=5.3$ $\mathrm{Hz}), 2.50(1 \mathrm{H}, \mathrm{m}), 2.23-1.94(6 \mathrm{H}$, series of $\mathrm{m}), 1.08(3 \mathrm{H}$, s), $1.07(3 \mathrm{H}, \mathrm{s}) .{ }^{13} \mathbf{C} \mathbf{~ N M R}\left(75 \mathrm{MHz}, \mathrm{CDCl}_{3}\right)$ : $\square$ 138.3, 136.6, 133.5, 131.2, 71.5, 67.2, 58.6, 51.6, 48.4, 45.0, 41.9, 39.3, 30.9, 30.3, 28.6. IR ( NaCl, thin film): 3033, 2951, 2923, 2861, 2832, 1478, 1448, 1360, 1015, 883, $755 \mathrm{~cm}^{-1}$. GC-MS $(70 \mathrm{eV}) \mathrm{m} / \mathrm{z}$ (\% rel intensity): $216\left(\mathrm{M}^{+}\right.$, 59), 201 (11), 186 (25), 171 (43), 159 (18), 143 (45), 129 (97), 115 (80), 106 (84), 91 (100), 77 (76), 65 (41), 55 (47), 39 (98). Anal. Calcd. for $\mathrm{C}_{15} \mathrm{H}_{20} \mathrm{O}: \mathrm{C}, 83.23$; $\mathrm{H}$, 9.26. Found: C, 83.63; H, 9.52.

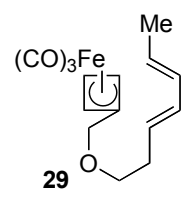

${ }^{1} \mathbf{H}$ NMR (400 MHz, $\left.\mathrm{CDCl}_{3}\right): \square 6.12-5.98$ $(2 \mathrm{H}, \mathrm{m}), 5.67-5.50(2 \mathrm{H}, \mathrm{m}), 4.14(2 \mathrm{H}, \mathrm{s}), 4.08(1 \mathrm{H}, \mathrm{s})$, $3.78(2 \mathrm{H}, \mathrm{s}), 3.50(2 \mathrm{H}, \mathrm{t}, J=7.0 \mathrm{~Hz}), 2.35(2 \mathrm{H}, \mathrm{q}, J=7.0$ $\mathrm{Hz}), 1.74(3 \mathrm{H}, \mathrm{d}, J=7.1 \mathrm{~Hz}) .{ }^{13} \mathbf{C} \mathbf{N M R}(100 \mathrm{MHz}$, $\left.\mathrm{CDCl}_{3}\right): \square 214.4,132.5,131.6,128.0,127.5,80.3,70.5$, 66.5, 65.0, 33.1, 18.2. IR ( NaCl, thin film): 3018, 2914, $2856,2045,1966,1352,1091,989,614,589 \mathrm{~cm}^{-1}$. GCMS (70 eV) mlz (\% rel intensity): $316\left(\mathrm{M}^{+}, 3\right), 288(0.3)$, 260 (29), 232 (49), 200 (27), 174 (23), 148 (84), 134 (90), 121 (100), 95 (99), 81 (75), 56 (88), 39 (45). Anal. Calcd. for $\mathrm{C}_{15} \mathrm{H}_{16} \mathrm{O}_{4} \mathrm{Fe}$ : C, 56.96; $\mathrm{H}, 5.06$. Found: C, 56.99; H, 5.10.

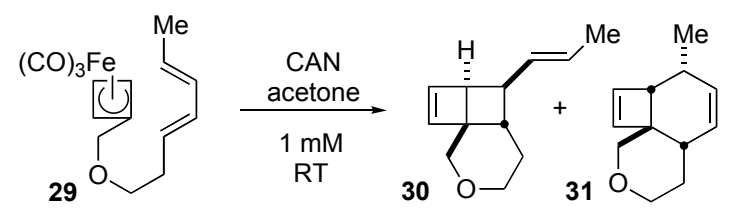

A solution of diene ether $29(78 \mathrm{mg}, 0.24 \mathrm{mmol}, 1.0$ equiv) and CAN (650 mg, $1.19 \mathrm{mmol}, 5.0$ equiv) in acetone $(240 \mathrm{~mL})$ was treated according to the general cycloaddition procedure (method A) and purified by $\mathrm{SiO}_{2}$ gel flash column chromatography (40:1 pentanes: $\left.\mathrm{Et}_{2} \mathrm{O}\right)$ to afford the two inseparable cycloadducts $(\mathbf{3 0 : 3 1}=1: 12,32$ $\mathrm{mg}, 75 \%)$, which can all be converged to the [4+2] cycloadduct $\mathbf{3 1}$ upon subjection to the general thermal isomerization procedure.

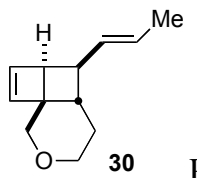
$\left.\mathrm{CDCl}_{3}\right): \square 6.25(1 \mathrm{H}, \mathrm{d},=2.4 \mathrm{~Hz}), 6.10(1 \mathrm{H}, \mathrm{J}, \mathrm{H}=2.4 \mathrm{~Hz})$, $3.32(1 \mathrm{H}, \mathrm{dd}, J=11.6,3.2 \mathrm{~Hz}), 3.27(1 \mathrm{H}, \mathrm{dd}, J=7.6,2.0$ $\mathrm{Hz}), 2.60(1 \mathrm{H}, \mathrm{m}) .{ }^{13} \mathrm{C}$ NMR $\left(75 \mathrm{MHz}, \mathrm{CDCl}_{3}\right): \mathrm{d} 140.8$, 139.4, 133.1, 124.8, 69.2, 64.5, 48.9, 44.2, 36.1, 30.8, 18.1 . 
<smiles>C[C@H]1C=C[C@H]2CCOCC23C=C[C@@H]13</smiles>

$31{ }^{1} \mathbf{H}$ NMR (400 MHz, $\left.\mathrm{CDCl}_{3}\right): \square 6.32(1 \mathrm{H}, \mathrm{d}, J=$ $2.9 \mathrm{~Hz}), 6.01(1 \mathrm{H}, \mathrm{d}, J=2.9 \mathrm{~Hz}), 5.50(1 \mathrm{H}, \mathrm{dt}, J=9.2,2.7$ $\mathrm{Hz}), 5.41(1 \mathrm{H}$, ddt, $J=9.2,3.0,0.8 \mathrm{~Hz}), 4.01(1 \mathrm{H}, \mathrm{dt}, J=$ $11.2,3.2 \mathrm{~Hz}), 3.80(1 \mathrm{H}, \mathrm{d}, J=10.8 \mathrm{~Hz}), 3.59(1 \mathrm{H}, \mathrm{d}, J=$ $10.8 \mathrm{~Hz}), 3.48(1 \mathrm{H}, \mathrm{ddd}, J=11.2,8.8,6.0 \mathrm{~Hz}), 2.45(1 \mathrm{H}$, d, $J=5.6 \mathrm{~Hz}), 2.26(1 \mathrm{H}, \mathrm{m}), 2.06(1 \mathrm{H}, \mathrm{m}), 1.66-1.59(2 \mathrm{H}$, series of m), $1.02(3 \mathrm{H}, \mathrm{d}, J=7.3 \mathrm{~Hz}) .{ }^{13} \mathbf{C ~ N M R}(75 \mathrm{MHz}$, $\left.\mathrm{CDCl}_{3}\right): \square 139.2,134.0,132.4,130.2,75.8,69.1,52.5$, 49.3, 39.4, 32.0, 29.4, 18.2. IR ( NaCl, thin film): 3016 , 2955, 2928, 2884, 2831, 1460, 1386, 1139, 1093, $774 \mathrm{~cm}^{-}$ 1. GC-MS $(70 \mathrm{eV}) \mathrm{m} / \mathrm{z}$ (\% rel intensity): $176\left(\mathrm{M}^{+}, 13\right)$, 161 (27), 147 (34), 131 (97), 117 (97), 105 (97), 91 (100), 77 (87), 65 (50), 51 (47), 39 (93). Anal. Calcd. for $\mathrm{C}_{12} \mathrm{H}_{16} \mathrm{O}: \mathrm{C}, 81.81 ; \mathrm{H}, 9.09$. Found: C, 81.41; H, 8.86.

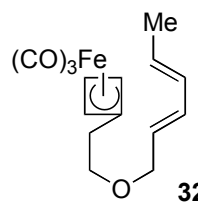

$32{ }^{1} \mathbf{H}$ NMR (400 MHz, $\left.\mathrm{CDCl}_{3}\right): \square 6.21(1 \mathrm{H}$, $\mathrm{dd}, J=16.0,11.0 \mathrm{~Hz}), 6.06(1 \mathrm{H}, \mathrm{m}), 5.72(1 \mathrm{H}, \mathrm{dd}, J=15.0$, $7.0 \mathrm{~Hz}), 5.61(1 \mathrm{H}, \mathrm{dt}, J=15.0,7.0 \mathrm{~Hz}), 4.03(2 \mathrm{H}, \mathrm{s}), 4.00$ $(1 \mathrm{H}, \mathrm{s}), 3.98(2 \mathrm{H}, \mathrm{d}, J=6.4 \mathrm{~Hz}), 3.44(2 \mathrm{H}, \mathrm{t}, J=6.4 \mathrm{~Hz})$, $2.25(3 \mathrm{H}, \mathrm{t}, J=6.4 \mathrm{~Hz}), 1.76(3 \mathrm{H}, \mathrm{d}, J=6.6 \mathrm{~Hz}) .{ }^{13} \mathbf{C}$ NMR (100 MHz, $\left.\mathrm{CDCl}_{3}\right)$ : $\square$ 215.2, 133.5, 131.0, 130.4, 126.6, 85.7, 71.5, 69.7, 64.9, 60.8, 28.0, 18.3. IR $(\mathrm{NaCl}$, thin film): 3018, 2914, 2855, 2031, 1992, 1365, 1104, 995, 594, $619 \mathrm{~cm}^{-1}$. GC-MS $(70 \mathrm{eV}) \mathrm{mlz}(\%$ rel intensity): $288\left(\mathrm{M}^{+}-1 \mathrm{CO}, 16\right), 260\left(\mathrm{M}^{+}-2 \mathrm{CO}, 27\right), 232$ (M+3CO, 56), 200 (31), 174 (22), 160 (28), 148 (82), 134 (100), 108 (11), 95 (58), 81 (60), 56 (83), 41 (51). Anal. Calcd. for $\mathrm{C}_{15} \mathrm{H}_{16} \mathrm{O}_{4} \mathrm{Fe}$ : C, 56.96; H, 5.06. Found: $\mathrm{C}$, 57.20; H, 5.25.

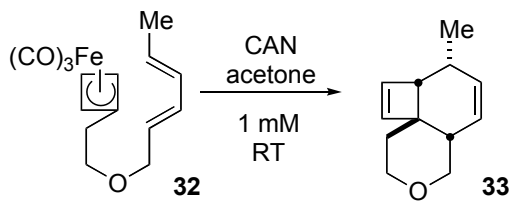

A solution of diene ether 32 (140 mg, $0.44 \mathrm{mmol}, 1.0$ equiv) and CAN (1.09 g, $1.99 \mathrm{mmol}, 4.5$ equiv) in acetone $(450 \mathrm{~mL})$ was treated according to the general cycloaddition procedure (method $\mathrm{A}$ ) and purified by $\mathrm{SiO}_{2}$ gel flash column chromatography (40:1 pentanes: $\left.\mathrm{Et}_{2} \mathrm{O}\right)$ to give only [4+2] cycloadduct $\mathbf{3 3}$ as colorless oil (47 $\mathrm{mg}$, $60 \%) .{ }^{1} \mathbf{H}$ NMR $\left(400 \mathrm{MHz}, \mathrm{CDCl}_{3}\right): \square 6.33(1 \mathrm{H}, \mathrm{dd}, J=$ 2.9, $0.7 \mathrm{~Hz}), 6.04(1 \mathrm{H}, \mathrm{d}, J=2.9 \mathrm{~Hz}), 5.45(1 \mathrm{H}, \mathrm{ddt}, J=$ 9.2, 2.8, 1.1 Hz), $5.34(1 \mathrm{H}, \mathrm{dt}, J=9.2,2.9 \mathrm{~Hz}), 3.93(1 \mathrm{H}$, ddd, $J=11.4,4.4,1.1 \mathrm{~Hz}), 3.89(1 \mathrm{H}, \mathrm{dd}, J=11.0,4.0 \mathrm{~Hz})$, $3.54(1 \mathrm{H}$, ddd, $J=12.8,11.7,2.6 \mathrm{~Hz}), 3.30(1 \mathrm{H}, \mathrm{dd}, J=$ $11.7,11.0 \mathrm{~Hz}), 2.51(1 \mathrm{H}, \mathrm{d}, J=5.5 \mathrm{~Hz}), 2.31(1 \mathrm{H}, \mathrm{m}), 2.18$ $(1 \mathrm{H}, \mathrm{m}), 2.00(1 \mathrm{H}, \mathrm{dt}, J=12.6,4.4 \mathrm{~Hz}), 1.60(1 \mathrm{H}, \mathrm{dt}, J=$ 12.8, $1.8 \mathrm{~Hz}), 1.02(3 \mathrm{H}, \mathrm{d}, J=7.3 \mathrm{~Hz}),{ }^{13} \mathbf{C}$ NMR $(75$
$\left.\mathrm{MHz}, \mathrm{CDCl}_{3}\right)$ : $\square 137.5,134.5,133.3,125.8,68.7,66.4$, 53.1, 51.0, 41.5, 36.0, 31.9, 18.0. IR ( NaCl, thin film): 3022, 2956, 2928, 2908, 2841, 1456, 1373, 1213, 1158, 1102, 1079, 1010, 964, 847, 818, 772, 730, $619 \mathrm{~cm}^{-1}$. GC-MS $(70 \mathrm{eV}) \mathrm{m} / \mathrm{z}$ (\% rel intensity): $176\left(\mathrm{M}^{+}, 2\right), 161$ (6), 147 (10), 131 (55), 117 (59), 104 (63), 91 (100), 77 (40), 65 (24), 51 (28), 39 (63). Anal. Calcd. for $\mathrm{C}_{12} \mathrm{H}_{16} \mathrm{O}$ : C, 81.82; H, 9.09. Found: C, 81.52; H, 8.98.

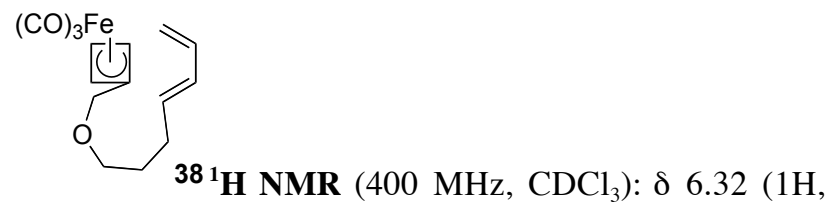
$\mathrm{dt}, J=17.2,10.5 \mathrm{~Hz}), 6.08(1 \mathrm{H}, \mathrm{dd}, J=15.4,10.1 \mathrm{~Hz})$, $5.70(1 \mathrm{H}, \mathrm{dt}, J=15.4,7.3 \mathrm{~Hz}), 5.10(1 \mathrm{H}, \mathrm{ddd}, J=17.2,0.6$, $0.5 \mathrm{~Hz}), 4.98(1 \mathrm{H}$, ddd, $J=10.1,1.1,0.6 \mathrm{~Hz}), 4.14(2 \mathrm{H}, \mathrm{s})$, $4.08(1 \mathrm{H}, \mathrm{s}), 3.76(2 \mathrm{H}, \mathrm{s}), 3.48(2 \mathrm{H}, \mathrm{t}, J=6.6 \mathrm{~Hz}), 2.17$ $(2 \mathrm{H}$, app q, $J=7.3 \mathrm{~Hz}), 1.69(2 \mathrm{H}, \mathrm{tt}, J=7.3,6.6 \mathrm{~Hz}) .{ }^{13} \mathrm{C}$ NMR (100 MHz, $\left.\mathrm{CDCl}_{3}\right): \square 214.5,137.3,134.6,131.7$, $115.3,87.5,70.2,66.5,64.9,62.7,29.3,29.2$. IR $(\mathrm{NaCl}$, thin film): $3075,3030,3000,2940,2855,2045,1970$, 1650, 1600, 1443, 1350, 1098, 1005, 900, 614, $591 \mathrm{~cm}^{-1}$. GC-MS $(70 \mathrm{eV}) \mathrm{m} / \mathrm{z}$ (\% rel intensity): $316\left(\mathrm{M}^{+}, 16\right), 288$ $\left(\mathrm{M}^{+}-1 \mathrm{CO}, 1\right), 260\left(\mathrm{M}^{+}-2 \mathrm{CO}, 8\right), 232\left(\mathrm{M}^{+}-3 \mathrm{CO}, 100\right), 205$ (18), 174 (12), 166 (30), 148 (27), 134 (22), 121 (21), 95 (21), 81 (14), 56 (23), 41 (15). Anal. Calcd. for $\mathrm{C}_{15} \mathrm{H}_{16} \mathrm{O}_{4} \mathrm{Fe}$ : C, 56.96; H, 5.06. Found: C, 57.06; H, 5.13.

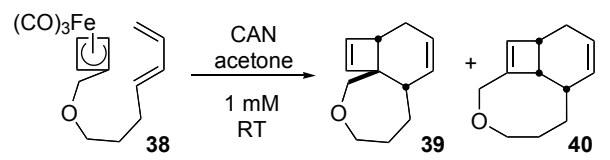

A solution of diene ether $\mathbf{3 8}(180 \mathrm{mg}, 0.633 \mathrm{mmol}, 1.0$ equiv) and TMAO (950 mg, $12.66 \mathrm{mmol}, 20$ equiv) in acetone $(630 \mathrm{~mL}, 1 \mathrm{mM})$ was treated according to the general cycloaddition procedure $(\operatorname{method} \mathrm{B})$ and purified by $\mathrm{SiO}_{2}$ gel gradient flash column chromatography (40:1; 20:1 pentanes: $\left.\mathrm{Et}_{2} \mathrm{O}\right)$ to afford two endo-[4+2] cycloadducts as colorless oils $(\mathbf{3 9 : 4 0}=2.5: 1.0,28 \%)$. The endo relative stereochemistry in the cycloadducts was assigned by NOE analysis.

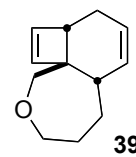

$39{ }^{1} \mathbf{H}$ NMR (500 MHz, $\left.\mathrm{CDCl}_{3}\right): \square 6.25(1 \mathrm{H}, \mathrm{d}, J=$ $2.9 \mathrm{~Hz}), 5.98(1 \mathrm{H}, \mathrm{dd}, J=2.9,1.0 \mathrm{~Hz}), 5.70(1 \mathrm{H}, \mathrm{ddd}, J=$ 12.7, 5.9, $3.9 \mathrm{~Hz}), 5.50(1 \mathrm{H}, \mathrm{dm} J=9.3 \mathrm{~Hz}), 3.80(1 \mathrm{H}, \mathrm{d}$, $J=11.7 \mathrm{~Hz}), 3.75(1 \mathrm{H}, \mathrm{d}, J=11.7 \mathrm{~Hz}), 3.80-3.70(2 \mathrm{H}, \mathrm{m})$, $2.52(1 \mathrm{H}, \mathrm{dd}, J=4.4,3.4 \mathrm{~Hz}), 2.26(1 \mathrm{H}, \mathrm{dm}, J=12.7 \mathrm{~Hz})$, 2.10-2.08 (2H, m), 1.90-1.75 (3H, m), 1.60-1.54 (1H, m). ${ }^{13}$ C NMR $\left(125 \mathrm{MHz}, \mathrm{CDCl}_{3}\right)$ : $\square$ 138.5, 137.2, 133.1, 124.6, 78.6, 69.1, 55.2, 44.6, 40.6, 30.9, 29.3, 25.9. IR (NaCl, thin film): 3026, 2924, 2857, 2834, 1456, 1263, $1125,1073,835,752,707 \mathrm{~cm}^{-1}$. GC-MS $(70 \mathrm{eV}) \mathrm{mlz}(\%$ 
rel intensity): $176\left(\mathrm{M}^{+}, 1\right), 161$ (2), 147 (4), 135 (8), 129 (17), 117 (100), 105 (17), 91 (51), 77 (19), 65 (12), 51 (9), 39 (17). Anal. Calcd. for $\mathrm{C}_{12} \mathrm{H}_{16} \mathrm{O}: \mathrm{C}, 81.82 ; \mathrm{H}, 9.09$. Found: C, 81.71; H, 8.93.

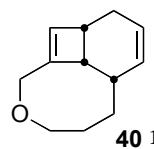

${ }^{1} \mathbf{H}$ NMR $\left(500 \mathrm{MHz}, \mathrm{CDCl}_{3}\right): \square 5.78(1 \mathrm{H}, \mathrm{ddt}$, $J=9.8,6.8,2.9 \mathrm{~Hz}), 5.72(1 \mathrm{H}, \mathrm{s}), 5.57(1 \mathrm{H}, \mathrm{dt}, J=9.8,2.4$ $\mathrm{Hz}), 4.25$ (1H, ddt, $J=16.1,3.9,1.5 \mathrm{~Hz}), 3.89(1 \mathrm{H}, \mathrm{dd}, J=$ $16.1,1.5 \mathrm{~Hz}), 3.68(1 \mathrm{H}$, ddd, $J=12.2,7.8,4.9 \mathrm{~Hz}), 3.48$ $(1 \mathrm{H}$, ddd, $J=12.2,6.3,4.4 \mathrm{~Hz}), 3.14(1 \mathrm{H}, \mathrm{dd}, J=5.4,4.9$ $\mathrm{Hz}), 2.91(1 \mathrm{H}, \mathrm{dm}, J=3.9 \mathrm{~Hz}), 2.49(1 \mathrm{H}$, app heptet, $J=$ $2.9 \mathrm{~Hz}), 2.11(1 \mathrm{H}, \mathrm{ddm}, J=16.1,6.8 \mathrm{~Hz}), 2.03(1 \mathrm{H}$, dhex, $J=16.1,2.9 \mathrm{~Hz}), 1.90(1 \mathrm{H}$, dddd, $J=14.1,6.8,3.9 \mathrm{~Hz})$, 1.78-1.62 (3H, m). ${ }^{13} \mathbf{C}$ NMR $\left(125 \mathrm{MHz}, \mathrm{CDCl}_{3}\right)$ : 148.1, 130.0, 129.8, 126.2,70.2, 67.3, 46.6, 37.3, 35.5, 28.0, 26.7, 24.5. IR ( NaCl, thin film): 3027, 2910, 2830, 1448, 1350, 1164, 1108, 1090, 1014, 890, 814, $620 \mathrm{~cm}^{-1}$. GC-MS $(70 \mathrm{eV}) \mathrm{m} / \mathrm{z}$ (\% rel intensity): $176\left(\mathrm{M}^{+}, 5\right), 161$ (5), 147 (10), 135 (21), 129 (21), 117 (13), 105 (36), 91 (100), 79 (45), 65 (20), 51 (17), 39 (39). Anal. Calcd. for $\mathrm{C}_{12} \mathrm{H}_{16} \mathrm{O}$ : C, 81.82; H, 9.09. Found: C, 81.60; H, 9.05.

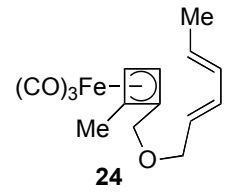

${ }^{1}$ H NMR (400 MHz, $\left.\mathrm{CDCl}_{3}\right)$ : $\square 6.20(1 \mathrm{H}$, $\mathrm{dd}, J=15.2,10.4 \mathrm{~Hz}), 6.06(1 \mathrm{H}, \mathrm{ddm}, J=14.8,10.4 \mathrm{~Hz})$, $5.71(1 \mathrm{H}, \mathrm{dq}, J=14.8,6.8 \mathrm{~Hz}), 5.60(1 \mathrm{H}$, app dt, $J=15.2$, $6.0 \mathrm{~Hz}), 4.12(1 \mathrm{H}, \mathrm{s}), 4.05(1 \mathrm{H}, \mathrm{s}), 4.02(2 \mathrm{H}, \mathrm{d}, J=6.0$ $\mathrm{Hz}), 3.87(1 \mathrm{H}, \mathrm{dt}, J=12.4 \mathrm{~Hz}), 3.75(1 \mathrm{H}, \mathrm{d}, J=12.4 \mathrm{~Hz})$, $1.81(3 \mathrm{H}, \mathrm{s}), 1.75(3 \mathrm{H}, \mathrm{d}, J=6.8 \mathrm{~Hz}),{ }^{13} \mathbf{C}$ NMR $(100$ $\left.\mathrm{MHz}, \mathrm{CDCl}_{3}\right)$ : $\square 214.6,133.7,130.8,130.5,126.4,86.7$, $80.9,71.3,64.6,64.4,60.1,18.5,12.4$. IR $(\mathrm{NaCl}$, thin film): 3021, 2916, 2864, 2040, 1957, 1448, 1373, 1347, 1107, 1078, 1059, 990, 618, $600 \mathrm{~cm}^{-1}$. GC-MS $(70 \mathrm{eV})$ $m l z$ (\% rel intensity): $316\left(\mathrm{M}^{+}, 12\right), 288\left(\mathrm{M}^{+}-1 \mathrm{CO}, 0.4\right)$, $260\left(\mathrm{M}^{+}-2 \mathrm{CO}, 49\right), 232\left(\mathrm{M}^{+}-3 \mathrm{CO}, 100\right), 212$ (7), 200 (12), 188 (6), 176 (10), 162 (18), 152 (69), 134 (52), 121 (17), 110 (15), 95 (47), 81 (44), 56 (77), 41 (37). Anal. Calcd. for $\mathrm{C}_{15} \mathrm{H}_{16} \mathrm{O}_{4} \mathrm{Fe}: \mathrm{C}, 56.96 ; \mathrm{H}, 5.06$. Found: C, 57.07; H, 5.06 .

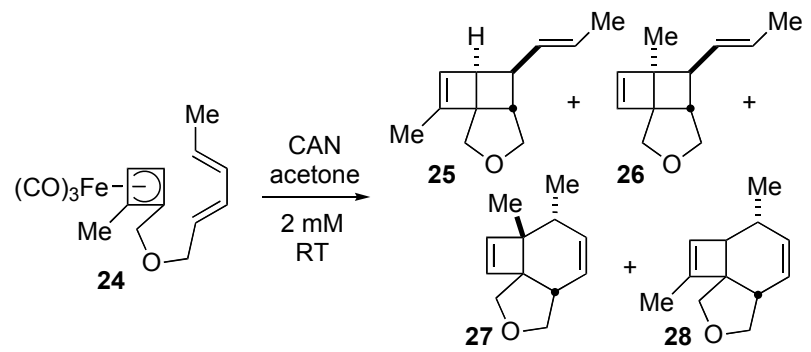

A solution of diene ether $24(150 \mathrm{mg}, 0.47 \mathrm{mmol}, 1.0$ equiv) and CAN (1.3 g, $2.37 \mathrm{mmol}, 5.0$ equiv) in acetone
$(470 \mathrm{~mL})$ was treated according to the general cycloaddition procedure (method A). ${ }^{1} \mathrm{H}$ NMR analysis of the crude reaction mixture suggested a formation of all possible cycloadducts: two regioisomeric [2+2] and two regioisomeric [4+2] cycloadducts $(\mathbf{2 5 : 2 6 : 2 7 : 2 8}=28: 10: 3: 8)$. Upon purification of the crude by $\mathrm{SiO}_{2}$ gel gradient flash column chromatography $\left(100: 1 ; 40: 1\right.$ pentanes: $\left.\mathrm{Et}_{2} \mathrm{O}\right)$, three fractions were collected in the following order: minor [2+2] regioisomer $26(12 \mathrm{mg})$, major [2+2] regioisomer $25(34 \mathrm{mg})$, and a mixture of the [4+2] regioisomers $(\mathbf{2 8 : 2 7}=8: 3,12 \mathrm{mg})$, yielding a total of 58 $\mathrm{mg}$ of cycloadducts $(70 \%)$. Subjection of the [2+2] cycloadducts 25 and 26 independently to the general thermal isomerization conditions afforded the corresponding [4+2] cycloadducts $\mathbf{2 7}$ and 28, respectively, suggesting that a [3.3] sigmatropic rearrangement (Cope) was operative during the isomerization.

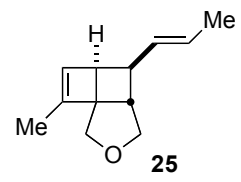

$\mathrm{dd}, J=0.9,0.6 \mathrm{~Hz}), 5.38(1 \mathrm{H}, \mathrm{dd}, J=7.2,5.4 \mathrm{~Hz}), 5.36$ $(1 \mathrm{H}, \mathrm{dq}, J=7.2,5.7 \mathrm{~Hz}), 3.95(1 \mathrm{H}, \mathrm{ddd}, J=9.6,0.9,0.6$ $\mathrm{Hz}), 3.77(1 \mathrm{H}, \mathrm{d}, J=9.6 \mathrm{~Hz}), 3.75(1 \mathrm{H}, \mathrm{dd}, J=9.6,6.0$ $\mathrm{Hz}), 3.56(1 \mathrm{H}, \mathrm{d}, J=9.6 \mathrm{~Hz}), 2.98(1 \mathrm{H}, \mathrm{d}, J=7.8 \mathrm{~Hz}), 2.68$ $(1 \mathrm{H}, \mathrm{dt}, J=7.8,5.4 \mathrm{~Hz}), 2.27(1 \mathrm{H}$, app t, $J=5.4 \mathrm{~Hz}), 1.74$ $(3 \mathrm{H}, \mathrm{dd}, J=1.8,1.5 \mathrm{~Hz}), 1.63(3 \mathrm{H}, \mathrm{d}, J=5.7 \mathrm{~Hz}) .{ }^{13} \mathrm{C}$ NMR $\left(75 \mathrm{MHz}, \mathrm{CDCl}_{3}\right)$ : $\square$ 147.5, 133.1, 129.5, 124.7, 73.8, 69.2, 58.8, 46.1, 45.7, 43.3, 18.1, 13.6. IR $(\mathrm{NaCl}$, thin film): $3030,2954,2914,2845,1617,1437,1373$, 1064, 966, 913, $716 \mathrm{~cm}^{-1}$. GC-MS $(70 \mathrm{eV}) \mathrm{mlz}$ (\% rel intensity): $176\left(\mathrm{M}^{+}, 1\right), 161$ (6), 147 (17), 131 (100), 115 (38), 105 (55), 91 (100), 77 (62), 65 (34), 51 (38), 39 (78). Anal. Calcd. for $\mathrm{C}_{12} \mathrm{H}_{16} \mathrm{O}: \mathrm{C}, 81.82 ; \mathrm{H}, 9.09$. Found: $\mathrm{C}$, 81.44; H, 9.20.

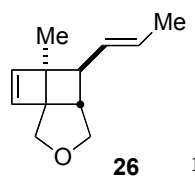

${ }^{1}$ H NMR (300 MHz, $\left.\mathrm{CDCl}_{3}\right): \square 6.27(1 \mathrm{H}, \mathrm{d}$, $J=2.4 \mathrm{~Hz}), 6.19(1 \mathrm{H}, \mathrm{d}, J=2.4 \mathrm{~Hz}), 5.38(1 \mathrm{H}, \mathrm{m}), 5.39$ $(1 \mathrm{H}, \mathrm{t}, J=5.6 \mathrm{~Hz}), 3.87(1 \mathrm{H}, \mathrm{dd}, J=9.6,1.5 \mathrm{~Hz}), 3.72(1 \mathrm{H}$, d, $J=10.2 \mathrm{~Hz}), 3.71(1 \mathrm{H}, \mathrm{dd}, J=9.6,6.3 \mathrm{~Hz}), 3.52(1 \mathrm{H}, \mathrm{d}$, $J=10.2 \mathrm{~Hz}), 2.33(1 \mathrm{H}, \mathrm{dt}, J=5.6,1.5 \mathrm{~Hz}), 2.24(1 \mathrm{H}, \mathrm{dd}$, $J=6.6,4.8 \mathrm{~Hz}), 1.64(3 \mathrm{H}, \mathrm{d}, J=5.4 \mathrm{~Hz}), 1.15(3 \mathrm{H}, \mathrm{s}) .{ }^{13} \mathbf{C}$ NMR $\left(75 \mathrm{MHz}, \mathrm{CDCl}_{3}\right)$ : $\square$ 142.5, 135.6, 132.3, 124.9, 73.0, 67.3, 59.7, 54.0, 48.9, 46.2, 18.1, 17.5. IR $(\mathrm{NaCl}$, thin film): 3024, 2953, 2915, 2855, 2845, 1449, 1036, 1024, 966, 908, $757 \mathrm{~cm}^{-1}$. GC-MS $(70 \mathrm{eV}) \mathrm{m} / \mathrm{z}$ (\% rel intensity): $176\left(\mathrm{M}^{+}, 2\right), 161$ (3), 147 (8), 131 (72), 115 (36), 105 (41), 91 (96), 77 (61), 65 (34), 51 (40), 39 (100). Anal. Calcd. for $\mathrm{C}_{12} \mathrm{H}_{16} \mathrm{O}: \mathrm{C}, 81.82 ; \mathrm{H}, 9.09$. Found: $\mathrm{C}$, 81.46; H, 9.11. 


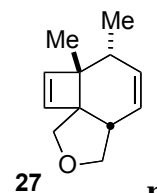

mp: $29.5-30.5{ }^{\circ} \mathrm{C} . \quad{ }^{\mathbf{1}} \mathbf{H}$ NMR $(300 \mathrm{MHz}$, $\left.\mathrm{CDCl}_{3}\right): \square 6.08(1 \mathrm{H}, \mathrm{d}, J=3.0 \mathrm{~Hz}), 5.94(1 \mathrm{H}, \mathrm{d}, J=3.0 \mathrm{~Hz})$, $5.79(1 \mathrm{H}, \mathrm{dt}, J=9.0,3.0 \mathrm{~Hz}), 5.52(1 \mathrm{H}, \mathrm{dt}, J=9.0,2.9 \mathrm{~Hz})$, $4.07(1 \mathrm{H}, \mathrm{dd}, J=7.5,7.2 \mathrm{~Hz}), 3.85(1 \mathrm{H}, \mathrm{d}, J=7.5 \mathrm{~Hz})$, $3.72(1 \mathrm{H}, \mathrm{d}, J=7.5 \mathrm{~Hz}), 3.49(1 \mathrm{H}, \mathrm{dd}, J=12.3,7.2 \mathrm{~Hz})$, $2.52(1 \mathrm{H}$, dddd, $J=12.3,7.5,5.1,2.7 \mathrm{~Hz}), 2.05(1 \mathrm{H}$, ddq, $J=7.2,4.5,2.7 \mathrm{~Hz}), 1.16(3 \mathrm{H}, \mathrm{s}), 1.02(3 \mathrm{H}, \mathrm{d}, J=7.2 \mathrm{~Hz})$. ${ }^{13}$ C NMR (75 MHz, $\left.\mathrm{CDCl}_{3}\right)$ : $\square$ 138.0, 136.9, 136.7, 124.3, 71.1, 68.7, 59.9, 51.3, 44.0, 39.8, 18.6, 15.3. IR $(\mathrm{NaCl}$, thin film): 3029, 2957, 2927, 2856, 2825, 1455, 1371, $1107,1031,1001,977,889,771,705,623 \mathrm{~cm}^{-1}$. GC-MS $(70 \mathrm{eV}) \mathrm{mlz}$ (\% rel intensity): $176\left(\mathrm{M}^{+}, 0.4\right), 161$ (4), 146 (9), 131 (51), 115 (34), 105 (29), 91 (71), 77 (56), 65 (31), 51 (43), 39 (100). Anal. Calcd. for $\mathrm{C}_{12} \mathrm{H}_{16} \mathrm{O}: \mathrm{C}, 81.81 ; \mathrm{H}$, 9.09. Found: C, 81.68; H, 8.92.

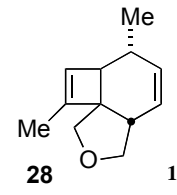

${ }^{1} \mathbf{H}$ NMR $\left(300 \mathrm{MHz}, \mathrm{CDCl}_{3}\right)$ : $\square 5.80(1 \mathrm{H}, \mathrm{dt}$, $J=8.7,3.2 \mathrm{~Hz}), 5.67(1 \mathrm{H}, \mathrm{m}), 5.52(1 \mathrm{H}, \mathrm{ddt}, J=9.0,2.7$, $0.9 \mathrm{~Hz}), 4.09(1 \mathrm{H}, \mathrm{t}, J=7.7 \mathrm{~Hz}), 3.94(1 \mathrm{H}, \mathrm{d}, J=7.5 \mathrm{~Hz})$, $3.85(1 \mathrm{H}, \mathrm{d}, J=7.5 \mathrm{~Hz}), 3.54(1 \mathrm{H}, \mathrm{dd}, J=12.5,7.5 \mathrm{~Hz})$, $2.67(1 \mathrm{H}, \mathrm{dm}, J=5.1 \mathrm{~Hz}), 2.55(1 \mathrm{H}, \mathrm{m}), 2.26(1 \mathrm{H}, \mathrm{m})$, $1.64(3 \mathrm{H}, \mathrm{dd}, J=1.8,1.5 \mathrm{~Hz}), 1.05(3 \mathrm{H}, \mathrm{d}, J=7.2 \mathrm{~Hz}) .{ }^{13} \mathrm{C}$ NMR (75 MHz, $\left.\mathrm{CDCl}_{3}\right)$ : $\square$ 149.1, 135.2, 125.9, 124.0, $70.5,68.4,58.5,45.6,44.0,33.9,17.5,14.5$. IR $(\mathrm{NaCl}$, thin film): 3028, 2957, 2928, 2904, 2870, 1436, 1044, 1007, 991, 885, $720 \mathrm{~cm}^{-1}$. GC-MS $(70 \mathrm{eV}) \mathrm{mlz}$ (\% rel intensity): $176\left(\mathrm{M}^{+}, 3\right), 161$ (7), 147 (18), 131 (100), 115 (26), 105 (44), 91 (79), 77 (39), 65 (22), 53 (23), 39 (46). Anal. Calcd. for $\mathrm{C}_{12} \mathrm{H}_{16} \mathrm{O}: \mathrm{C}, 81.82 ; \mathrm{H}, 9.09$. Found: $\mathrm{C}$, 81.61; H, 8.84.

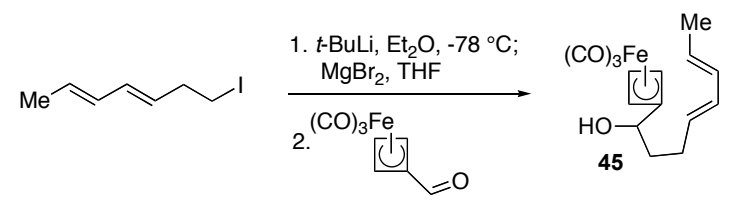

To a solution of 7-iodo-2,4(E,E)-heptadiene $(570 \mathrm{mg}, 2.57$ mmol, 1.0 equiv) in freshly distilled $\mathrm{Et}_{2} \mathrm{O}(6 \mathrm{~mL})$ at -78 ${ }^{\circ} \mathrm{C}$ under $\mathrm{N}_{2}$ was added a $1.80 \mathrm{M}$ solution of tert-butyllithium (3.28 mL, $5.91 \mathrm{mmol}, 2.3$ equiv). The resulting reaction mixture was stirred at $-78{ }^{\circ} \mathrm{C}$ for $15 \mathrm{~min}$, warmed to $0{ }^{\circ} \mathrm{C}$ for $15 \mathrm{~min}$, cooled back to $-78{ }^{\circ} \mathrm{C}$ and treated with a solution of $\mathrm{MgBr}_{2}(1.09 \mathrm{~g}, 5.91 \mathrm{mmol}, 2.3$ equiv $)$ in THF $(20 \mathrm{~mL})$. After stirring for $15 \mathrm{~min}$ at $-78{ }^{\circ} \mathrm{C}$ and at 0 ${ }^{\circ} \mathrm{C}$ for $0.5 \mathrm{~h}$, the reaction mixture was cooled back to -78 ${ }^{\circ} \mathrm{C}$ and treated with a solution of the iron aldehyde (282 $\mathrm{mg}, 1.28 \mathrm{mmol}, 0.5$ equiv) in THF ( $5 \mathrm{~mL})$. The resulting yellow-brown suspension was stirred at $-78{ }^{\circ} \mathrm{C}$ for $4 \mathrm{~h}$ at which no more starting aldehyde was evident as judged by TLC. The reaction mixture was quenched with wet $\mathrm{Et}_{2} \mathrm{O}$ and then transferred into a separatory funnel containing $\mathrm{Et}_{2} \mathrm{O}$ and sat' $\mathrm{NH}_{4} \mathrm{Cl}$ solution. The aqueous layer was separated and backextracted with $\mathrm{Et}_{2} \mathrm{O}$. The combined organic layer was washed with $\mathrm{H}_{2} \mathrm{O}$, brine, dried over $\mathrm{MgSO}_{4} / \mathrm{K}_{2} \mathrm{CO}_{3}$, filtered and concentrated in vacuo. The resulting residue was purified by $\mathrm{SiO}_{2}$ gel flash column chromatography $\left(9: 1\right.$ pentanes: $\left.\mathrm{Et}_{2} \mathrm{O}\right)$ to afford the diene alcohol 45 as yellow oil (260 mg, 65\%). ${ }^{\mathbf{1}} \mathbf{H}$ NMR (400 $\left.\mathrm{MHz}, \mathrm{CDCl}_{3}\right)$ : $\square$ 6.09-5.96 (2H, m), 5.65-5.48 (2H, m), $4.14(1 \mathrm{H}, \mathrm{s}), 4.10(1 \mathrm{H}, \mathrm{d}, J=9.2 \mathrm{~Hz}), 4.06(1 \mathrm{H}, \mathrm{dd}, J=$ $12.7,5.3 \mathrm{~Hz}), 4.03(1 \mathrm{H}, \mathrm{d}, J=9.2 \mathrm{~Hz}), 2.20(2 \mathrm{H}$, dpent, $J=$ $14.7,7.3 \mathrm{~Hz}), 1.72(3 \mathrm{H}, \mathrm{d}, J=6.6 \mathrm{~Hz}), 1.59(2 \mathrm{H}, \mathrm{q}, J=7.3$ $\mathrm{Hz}), 1.39(1 \mathrm{H}, \mathrm{d}, J=5.2 \mathrm{~Hz}) .{ }^{13} \mathbf{C}$ NMR $(100 \mathrm{MHz}$, $\left.\mathrm{CDCl}_{3}\right): \square 214.6,131.6,131.5,130.4,127.9,89.6,67.0$, $62.9,62.6,62.2,37.1,28.9,18.3$. IR $(\mathrm{NaCl}$, thin film): 3398, 3016, 2915, 2856, 2041, 1991, 1444, 989, 617, 584 $\mathrm{cm}^{-1}$. Anal. Calcd. for $\mathrm{C}_{15} \mathrm{H}_{16} \mathrm{O}_{4} \mathrm{Fe}: \mathrm{C}, 56.96 ; 5.06$. Found: C, 57.22; H, 5.19.

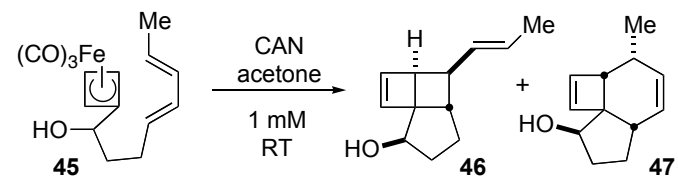

A solution of alcohol 45 (200 mg, $0.63 \mathrm{mmol}, 1.0$ equiv) and CAN (1.7 g, $3.16 \mathrm{mmol}, 5.0$ equiv) in acetone (630 $\mathrm{mL}$ ) was treated according to the general cycloaddition procedure (method A). ${ }^{1} \mathrm{H}$ NMR analysis of the crude reaction mixture suggested three cycloadducts were formed: only one [2+2] isomer and two diastereomeric [4+2] cycloadducts in 1:1.8:0.4 ratio, respectively. Purification of the crude by $\mathrm{SiO}_{2}$ gel gradient flash column chromatography $\left(10: 1 ; 4: 1\right.$ pentanes: $\left.\mathrm{Et}_{2} \mathrm{O}\right)$ gave the $[2+2]$ cycloadduct $46(30 \mathrm{mg}$, contaminated with a small amount of the minor [4+2] diastereomer) and the major $47[4+2]$ diastereomer cycloadduct as a white solid (50 mg, 72\% combined yield). The [2+2] cycloadduct 46 underwent a $[3,3]$ sigmatropic rearrangement to the corresponding major [4+2] diastereomer cycloadduct $\mathbf{4 7}$ upon subjection to the general thermal isomerization reaction condition.

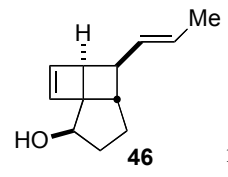
$\mathrm{dd}, J=2.6,1.5 \mathrm{~Hz}), 6.23(1 \mathrm{H}, \mathrm{d}, J=2.2 \mathrm{~Hz}), 5.46(1 \mathrm{H}$, ddq, $J=15.4,7.7,1.1 \mathrm{~Hz}), 5.35(1 \mathrm{H}, \mathrm{dq}, J=15.4,6.2 \mathrm{~Hz})$, $3.98(1 \mathrm{H}, \mathrm{d}, J=3.7 \mathrm{~Hz}), 2.90(1 \mathrm{H}, \mathrm{d}, J=7.7 \mathrm{~Hz}), 2.38(1 \mathrm{H}$, dt, $J=7.7,4.8 \mathrm{~Hz}), 2.26(1 \mathrm{H}, \mathrm{dd}, J=8.1,4.8 \mathrm{~Hz}), 2.07$ $(1 \mathrm{H}, \mathrm{tt}, J=12.8,7.7 \mathrm{~Hz}), 1.92(1 \mathrm{H}, \mathrm{ddt}, J=13.6,6.6,3.7$ $\mathrm{Hz}), 1.84(1 \mathrm{H}, \mathrm{dd}, J=13.6,7.3 \mathrm{~Hz}), 1.65(1 \mathrm{H}, \mathrm{d}, J=6.6$ $\mathrm{Hz}), 1.62(3 \mathrm{H}, \mathrm{d}, J=6.2 \mathrm{~Hz}) .{ }^{13} \mathbf{C}$ NMR $(100 \mathrm{MHz}$, $\left.\mathrm{CDCl}_{3}\right): \square 139.1,137.7,133.1,124.5,74.9,62.0,46.6$, 
43.4, 41.3, 34.5, 29.3, 18.3. IR ( NaCl, thin film): 3348 , 3027, 2940, 2858, 1439, 1306, 1127, 1016, 966, $746 \mathrm{~cm}^{-1}$. GC-MS (70 eV) $\mathrm{m} / \mathrm{z}$ (\% rel intensity): $176\left(\mathrm{M}^{+}, 14\right), 158$ $\left(\mathrm{M}^{+}-\mathrm{H}_{2} \mathrm{O}, 32\right), 143$ (64), 130 (87), 117 (87), 105 (36), 91 (100), 77 (65), 65 (33), 51 (40), 39 (81). Anal. Calcd. for $\mathrm{C}_{12} \mathrm{H}_{16} \mathrm{O}$ : C, 81.82; H, 9.09. Found: C, 81.60; H, 8.89.

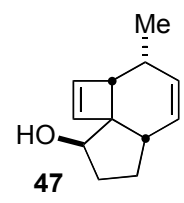

mp: $62-66{ }^{\circ} \mathrm{C} .{ }^{1} \mathbf{H}$ NMR $\left(400 \mathrm{MHz}, \mathrm{CDCl}_{3}\right)$ : $\square 6.02(1 \mathrm{H}, \mathrm{d}, J=2.9 \mathrm{~Hz}), 5.88(1 \mathrm{H}, \mathrm{dd}, J=2.9,1.1 \mathrm{~Hz})$, $5.85(1 \mathrm{H}, \mathrm{dt}, J=9.2,2.9 \mathrm{~Hz}), 5.38(1 \mathrm{H}, \mathrm{ddt}, J=9.2,2.8$, $1.0 \mathrm{~Hz}), 4.21(1 \mathrm{H}, \mathrm{d}, J=5.9 \mathrm{~Hz}), 2.97(1 \mathrm{H}, \mathrm{d}, J=5.5 \mathrm{~Hz})$, $2.60(1 \mathrm{H}$, ddt, $J=10.0,5.3,2.6 \mathrm{~Hz}), 2.39(1 \mathrm{H}$, ddq, $J=9.8$, 4.9, $2.6 \mathrm{~Hz}), 2.18(1 \mathrm{H}$, dddd, $J=17.0,10.8,5.9,2.6 \mathrm{~Hz})$, $1.94(1 \mathrm{H}$, ddt, $J=11.0,7.4,2.7 \mathrm{~Hz}), 1.66(1 \mathrm{H}, \mathrm{ddd}, J=$ $15.5,9.2,7.1 \mathrm{~Hz}), 1.28(1 \mathrm{H}, \mathrm{ddt}, J=12.6,11.1,7.1 \mathrm{~Hz})$, $1.38\left(1 \mathrm{H}\right.$, br s), $1.06(3 \mathrm{H}, \mathrm{d}, J=7.3 \mathrm{~Hz}),{ }^{13}$ C NMR (75 $\left.\mathrm{MHz}, \mathrm{CDCl}_{3}\right)$ : $\square$ 138.2, 134.6, 132.4, 129.4, 75.6, 64.1, $47.7,40.1,34.3,33.3,24.3,18.0$. IR ( NaCl, thin film): 3428 (br), 3029, 2948, 2869, 1686, 1390, 1226, 1119, 1031, 995, $798 \mathrm{~cm}^{-1}$. GC-MS $(70 \mathrm{eV}) \mathrm{mlz}(\%$ rel intensity): 176 (3), 158 (16), 143 (65), 130 (68), 117 (100), 105 (38), 91 (91), 77 (47), 65 (25), 51 (29), 39 (57). Anal. Calcd. for $\mathrm{C}_{12} \mathrm{H}_{16} \mathrm{O}: \mathrm{C}, 81.82 ; \mathrm{H}, 9.09$. Found: $\mathrm{C}$, 81.53; H, 9.00 .

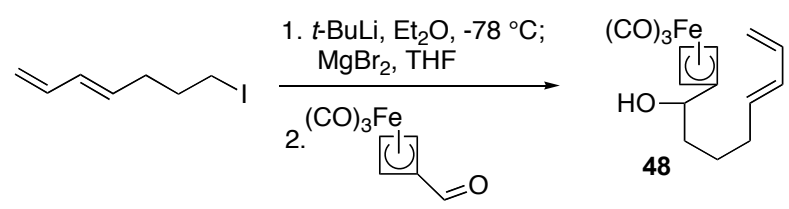

To a solution of 7-iodo-1,3(E,E)-heptadiene (444 mg, 2.0 mmol, 1.0 equiv) in freshly distilled $\mathrm{Et}_{2} \mathrm{O}(1.5 \mathrm{~mL})$ at -78 ${ }^{\circ} \mathrm{C}$ under $\mathrm{N}_{2}$ was added a $1.80 \mathrm{M}$ solution of tert-butyllithium (2.4 mL, $4.2 \mathrm{mmol}, 2.2$ equiv). The resulting mixture was stirred at $-78{ }^{\circ} \mathrm{C}$ for $20 \mathrm{~min}$ and at $0{ }^{\circ} \mathrm{C}$ for 10 min. After cooling back to $-78{ }^{\circ} \mathrm{C}$, the reaction mixture was treated with a solution of $\mathrm{MgBr}_{2}(920 \mathrm{mg}, 5.0$ mmol, 2.5 equiv) in THF $(15 \mathrm{~mL})$, stirred at $0{ }^{\circ} \mathrm{C}$ for 0.5 $\mathrm{h}$, cooled back to $-78{ }^{\circ} \mathrm{C}$ and then treated with a solution of iron aldehyde ( $220 \mathrm{mg}, 1.0 \mathrm{mmol}, 0.5$ equiv) in THF (5 $\mathrm{mL})$. After stirring at $-78{ }^{\circ} \mathrm{C}$ for $4 \mathrm{~h}$, the reaction mixture was quenched with wet $\mathrm{Et}_{2} \mathrm{O}$ and then transferred into a separatory funnel containing $\mathrm{Et}_{2} \mathrm{O}$ and sat'd $\mathrm{NH}_{4} \mathrm{Cl}$ solution. The aqueous layer was separated and backextracted with $\mathrm{Et}_{2} \mathrm{O}$. The combined organic layer was washed with $\mathrm{H}_{2} \mathrm{O}$, brine, dried over $\mathrm{MgSO}_{4} / \mathrm{K}_{2} \mathrm{CO}_{3}$, filtered and concentrated in vacuo. The resulting residue was purified by $\mathrm{SiO}_{2}$ gel flash column chromatography (9:1 pentanes: $\left.\mathrm{Et}_{2} \mathrm{O}\right)$ to afford the diene alcohol 48 as yellow oil (160 mg, 51\%). ${ }^{1} \mathbf{H}$ NMR (400 $\left.\mathrm{MHz} \mathrm{CDCl}_{3}\right)$ : $\square$ $6.31(1 \mathrm{H}, \mathrm{dt}, J=16.9,9.7 \mathrm{~Hz}), 6.07(1 \mathrm{H}$, ddt, $J=15.3$, $10.5,0.8 \mathrm{~Hz}), 5.69(1 \mathrm{H}, \mathrm{dt}, J=15.3,6.4 \mathrm{~Hz}), 5.10(1 \mathrm{H}$, ddd, $J=16.9,1.3,0.7 \mathrm{~Hz}$ ), 4.98 (1H, ddd, $J=10.5,1.2,0.7$
$\mathrm{Hz}), 4.14(1 \mathrm{H}, \mathrm{s}), 4.11(1 \mathrm{H}, \mathrm{d}, J=9.1 \mathrm{~Hz}), 4.04(1 \mathrm{H}, \mathrm{d}, J=$ $9.1 \mathrm{~Hz}), 4.03(1 \mathrm{H}, \mathrm{m}), 2.16-2.06(2 \mathrm{H}, \mathrm{m}), 1.64-1.42(4 \mathrm{H}$, series of $\mathrm{m}), 1.43(1 \mathrm{H}, \mathrm{d}, J=5.3 \mathrm{~Hz}),{ }^{13} \mathbf{C}$ NMR $(100$ $\left.\mathrm{MHz}, \mathrm{CDCl}_{3}\right)$ : $\square$ 215.1, 138.2, 135.5, 132.6, 116.2, 90.0, $68.3,63.7,63.4,63.0,37.9,33.2,26.2$. IR $(\mathrm{NaCl}$, thin film): 3421 (br), 2939, 2852, 2045, 1969, 1004, 613, 591 $\mathrm{cm}^{-1}$. Anal. Calcd. for $\mathrm{C}_{15} \mathrm{H}_{16} \mathrm{O}_{4} \mathrm{Fe}: \mathrm{C}, 56.96 ; \mathrm{H}, 5.06$. Found: C, 57.11; H, 5.16.

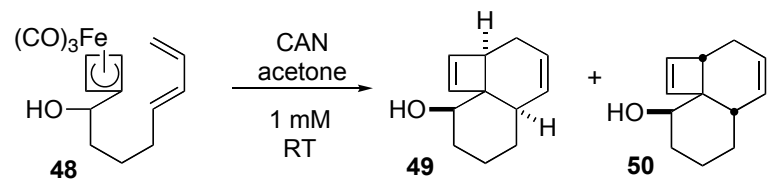

A solution of alcohol 48 (140 mg, $0.44 \mathrm{mmol}, 1.0$ equiv) and CAN (1.2 g, $2.2 \mathrm{mmol}, 5.0$ equiv) in acetone (440 $\mathrm{mL}$ ) was treated according to the general cycloaddition procedure (method A). Purification of the crude reaction mixture by $\mathrm{SiO}_{2}$ gel flash column chromatography (9:1 pentanes: $\left.\mathrm{Et}_{2} \mathrm{O}\right)$ afforded two diastereomeric [4+2] cycloadducts as colorless oils $(\mathbf{4 9 : 5 0}=2.4: 1,58 \mathrm{mg}, 74 \%)$.

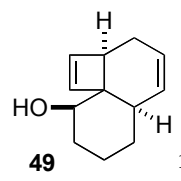

${ }^{1} \mathbf{H}$ NMR (400 MHz, $\left.\mathrm{CDCl}_{3}\right): \square 6.12(1 \mathrm{H}, \mathrm{d}$, $J=2.9 \mathrm{~Hz}), 5.98(1 \mathrm{H}, \mathrm{d}, J=2.9 \mathrm{~Hz}), 5.65(1 \mathrm{H}, \mathrm{ddd}, J=$ $13.1,5.1,3.3 \mathrm{~Hz}), 5.52(1 \mathrm{H}, \mathrm{dt}, J=9.1,2.4 \mathrm{~Hz}), 3.60(1 \mathrm{H}$, ddd, $J=11.4,8.4,4.4 \mathrm{~Hz}), 3.03(1 \mathrm{H}, \mathrm{dd}, J=4.0,3.7 \mathrm{~Hz})$, $2.08(2 \mathrm{H}, \mathrm{dt}, J=4.8,1.8 \mathrm{~Hz}), 1.97(1 \mathrm{H}, \mathrm{dm}, J=12.5 \mathrm{~Hz})$, $1.87(1 \mathrm{H}, \mathrm{dm}, J=13.5 \mathrm{~Hz}), 1.78(1 \mathrm{H}, \mathrm{m}), 1.67(1 \mathrm{H}, \mathrm{m})$, $1.65(1 \mathrm{H}, \mathrm{d}, J=8.4 \mathrm{~Hz}), 1.44(1 \mathrm{H}, \mathrm{tq}, J=13.2,4.0 \mathrm{~Hz})$, 1.33-1.19 (2H, m). ${ }^{13} \mathbf{C}$ NMR (100 $\left.\mathrm{MHz}, \mathrm{CDCl}_{3}\right)$ : 140.9, 132.6, 131.6, 125.0, 72.5, 59.7, 42.7, 40.7, 34.2, 28.9, 26.7, 24.8. IR ( $\mathrm{NaCl}$, thin film): 3390 (br), 3026, 2926, 2855, 1653, 1447, 1072, 1023, 765 cm-1. Anal. Calcd. for C12H16O: C, 81.82; H, 9.09. Found: C, 81.58; H, 9.12.

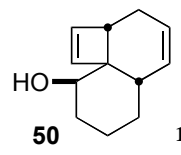

${ }^{1}$ H NMR (500 MHz, CDCl3): $\square 6.03(1 \mathrm{H}, \mathrm{d}$, $J=2.9 \mathrm{~Hz}), 5.98(1 \mathrm{H}, \mathrm{d}, \mathrm{J}=2.9 \mathrm{~Hz}), 5.63(1 \mathrm{H}, \mathrm{ddd}, J=$ $12.2,6.3,2.9 \mathrm{~Hz}), 5.54(1 \mathrm{H}, \mathrm{dt}, J=9.3,2.9 \mathrm{~Hz}), 3.95(1 \mathrm{H}$, $\mathrm{m}), 2.79(1 \mathrm{H}, \mathrm{dm}, J=5.9 \mathrm{~Hz}), 2.45(1 \mathrm{H}$, app dpent, $J=$ 12.7, $2.9 \mathrm{~Hz}), 2.19(1 \mathrm{H}$, dhex, $J=15.5,3.0 \mathrm{~Hz}), 2.12(1 \mathrm{H}$, ddd, $J=16.0,7.0,1.5 \mathrm{~Hz}), 1.80-1.56(5 \mathrm{H}, \mathrm{m}), 1.45(1 \mathrm{H}, \mathrm{d}$, $J=3.2 \mathrm{~Hz}), 1.32-1.26(1 \mathrm{H}, \mathrm{m}) .{ }^{13} \mathbf{C}$ NMR $(125 \mathrm{MHz}$, $\mathrm{CDCl} 3)$ : $\square 138.0,137.2,132.6,124.5,74.4,57.0,45.6$, $34.4,30.4,28.8,27.4,20.9$. IR ( NaCl, thin film): 3389 , 3027, 2926, 2856, 1657, 1446, 1351, 1128, 1072, 1023, 840, 765, 742, 704 cm-1. Anal. Calcd. for C12H16O: C, 81.82; H, 9.09. Found: C, 81.63; H, 9.12. 


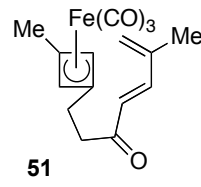
$\mathrm{d}, J=15.6 \mathrm{~Hz}), 6.17(1 \mathrm{H}, \mathrm{d}, J=15.6 \mathrm{~Hz}), 5.43(2 \mathrm{H}, \mathrm{d}, J=$ $7.3 \mathrm{~Hz}), 4.08(2 \mathrm{H}, \mathrm{s}), 2.68(2 \mathrm{H}, \mathrm{t}, J=7.3 \mathrm{~Hz}), 2.37(2 \mathrm{H}, \mathrm{t}$, $J=7.3 \mathrm{~Hz}), 1.92(3 \mathrm{H}, \mathrm{s}), 1.77(3 \mathrm{H}, \mathrm{s}),{ }^{13} \mathbf{C}$ NMR $(125$ $\left.\mathrm{MHz}, \mathrm{CDCl}_{3}\right)$ : $\square 215.5,199.1,145.6,141.0,126.8,125.8$, $82.2,82.0,66.0,40.4,21.7,18.3,13.2$. IR $(\mathrm{NaCl}$, thin film): 2976, 2952, 2921, 2034, 1948, 1691, 1666, 1624, 1598, 1439, 1371, 1260, 1173, 1048, 977, 910, 614, 590 $\mathrm{cm}^{-1}$. Anal. Calcd. for $\mathrm{C}_{16} \mathrm{H}_{16} \mathrm{O}_{4} \mathrm{Fe}: \mathrm{C}, 58.54 ; \mathrm{H}, 4.87$. Found: C, 58.49; H, 4.74.

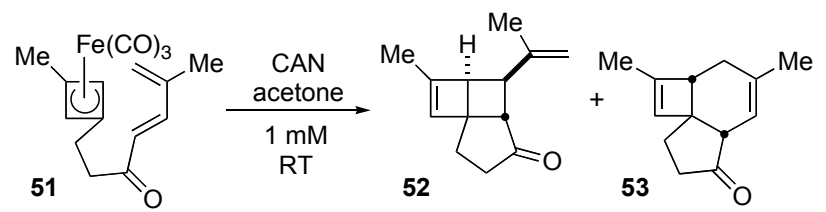

A solution of dienone 51 (150 mg, $0.46 \mathrm{mmol}, 1.0$ equiv) and TMAO (344 mg, $4.57 \mathrm{mmol}, 10$ equiv) in acetone (46 $\mathrm{mL}$ ) was treated according to the general cycloaddition procedure (method $\mathrm{B}$ ). The crude reaction mixture was purified by $\mathrm{SiO}_{2}$ gel flash column chromatography (29:1 pentanes: $\mathrm{Et}_{2} \mathrm{O}$ ) to give a mixture of $[2+2]$ and $[4+2]$ cycloadducts $(\mathbf{5 2 : 5 3 )}$, in approximately $1: 1$ ratio as determined by ${ }^{1} \mathrm{H}$ NMR analysis. The mixture of these cycloadducts was then charged with pentanes $(5 \mathrm{~mL})$ and the solution obtained was heated to $130{ }^{\circ} \mathrm{C}$ for $5 \mathrm{~h}$. The solvent was then removed in vacuo and the resulting residue was purified by $\mathrm{SiO}_{2}$ gel flash column chromatography (19:1 pentanes: $\left.\mathrm{Et}_{2} \mathrm{O}\right)$ to give only the endo cycloadduct 53 as colorless solid (15 mg, 17\%).

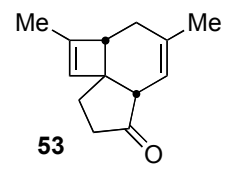
$\left.\mathrm{CDCl}_{3}\right): \square 5.65(1 \mathrm{H}, \mathrm{q}, J=1.5 \mathrm{~Hz}), 5.56(1 \mathrm{H}$, dhex, $J=7.7$, $1.5 \mathrm{~Hz}), 2.71(1 \mathrm{H}, \mathrm{d}, J=7.7 \mathrm{~Hz}), 2.55(1 \mathrm{H}, \mathrm{dm}, J=6.6$ $\mathrm{Hz}), 2.39$ (1H, dddd, $J=18.0,7.2,1.5,1.1 \mathrm{~Hz}), 2.18-2.94$ $(5 \mathrm{H}$, series of $\mathrm{m}), 1.74(3 \mathrm{H}, \mathrm{m}), 1.63(3 \mathrm{H}, \mathrm{dd}, J=1.5,1.1$ $\mathrm{Hz}) .{ }^{13} \mathbf{C}$ NMR (100 MHz, $\left.\mathrm{CDCl}_{3}\right)$ : $\square 218.8,146.7,134.7$, $130.4,116.5,53.5,50.3,48.2$, 38.8, 31.6, 30.6, 24.6, 14.0. IR ( $\mathrm{NaCl}$, thin film): $3031,2960,2908,1739,1435,1262$, 1123, 1040, $811 \mathrm{~cm}^{-1}$. H R M S : $\mathrm{m} / z$ calcd for $\left[\mathrm{C}_{13} \mathrm{H}_{16} \mathrm{ONa}\right]^{+}: 211.1099$. Found: 211.1089 (ES).

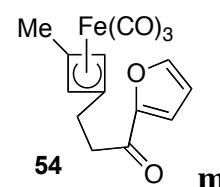

mp: $39-42{ }^{\circ} \mathrm{C} .{ }^{\mathbf{1}} \mathbf{H}$ NMR $\left(500 \mathrm{MHz}, \mathrm{CDCl}_{3}\right)$ : $7.61(1 \mathrm{H}, \mathrm{d}, J=1.5 \mathrm{~Hz}), 7.23(1 \mathrm{H}, \mathrm{d}, J=3.9 \mathrm{~Hz}), 6.57$ $(1 \mathrm{H}, \mathrm{dd}, J=3.9,1.5 \mathrm{~Hz}), 4.10(2 \mathrm{H}, \mathrm{s}), 2.92(2 \mathrm{H}, \mathrm{t}, J=7.1$
$\mathrm{Hz}), 2.45(2 \mathrm{H}, \mathrm{t}, J=7.1 \mathrm{~Hz}), 1.76(3 \mathrm{H}, \mathrm{s}) .{ }^{13} \mathbf{C}$ NMR $(100$ $\left.\mathrm{MHz}, \mathrm{CDCl}_{3}\right)$ : $\square 215.1,187.6,152.6,146.6,117.3,112.5$, $82.1,81.7,66.0,38.5,21.8,13.4$. IR $(\mathrm{NaCl}$, thin film): 3139, 3120, 3091, 2972, 2949, 2924, 2914, 2028, 1940, 1668, 1469, 1438, 1365, 773, 600, $587 \mathrm{~cm}^{-1}$. Anal. Calcd. for $\mathrm{C}_{15} \mathrm{H}_{12} \mathrm{O}_{5} \mathrm{Fe}$ : C, 54.88; $\mathrm{H}, 3.66$. Found: C, 54.89; H, 3.52.
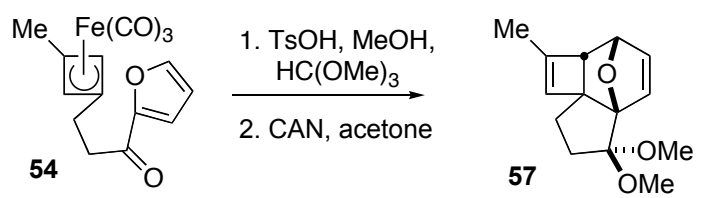

To a solution of furanyl ketone $54(210 \mathrm{mg}, 0.64 \mathrm{mmol}$, 1.0 equiv) in $\mathrm{MeOH}(6 \mathrm{~mL})$ and trimethylorthoformate (2 $\mathrm{mL}$ ) under $\mathrm{N}_{2}$ at RT was added $\mathrm{TsOH}$ (73 $\mathrm{mg}, 0.38 \mathrm{mmol}$, 0.6 equiv) all at once as a solid. The resulting reaction mixture was stirred at RT for $12 \mathrm{~h}$ at which no more starting ketone was evident as judged by TLC and GC. The reaction mixture was transferred into a separatory funnel containing cold $\mathrm{Et}_{2} \mathrm{O}$ and cold sat'd $\mathrm{NaHCO}_{3}$ solution. The aqueous layer was separated and backwashed with cold $\mathrm{Et}_{2} \mathrm{O}(2 \mathrm{x})$. The combined organic layer was washed with cold $\mathrm{H}_{2} \mathrm{O}$, brine, dried over $\mathrm{K}_{2} \mathrm{CO}_{3} / \mathrm{MgSO}_{4}$, filtered and concentrated in vacuo to give the corresponding dimethyl ketal $\mathrm{C}_{4} \mathrm{H}_{4} \mathrm{Fe}(\mathrm{CO})_{3}$ complex as yellow oil, which was used directly for cycloaddition without further purification. Partial characterization: ${ }^{1} \mathbf{H}$ NMR $(400 \mathrm{MHz}$, $\left.\mathrm{CDCl}_{3}\right): \square 7.39(1 \mathrm{H}, \mathrm{dd}, J=1.8,0.7 \mathrm{~Hz}), 6.41(1 \mathrm{H}, \mathrm{dd}, J=$ $3.3,0.7 \mathrm{~Hz}), 6.34(1 \mathrm{H}, \mathrm{dd}, J=3.3,1.8 \mathrm{~Hz}), 3.99(2 \mathrm{H}, \mathrm{s})$, $3.14(6 \mathrm{H}, \mathrm{s}), 1.98(2 \mathrm{H}, \mathrm{m}), 1.79(2 \mathrm{H}, \mathrm{m}), 1.72(3 \mathrm{H}, \mathrm{s})$. IR ( $\mathrm{NaCl}$, thin film): 2959, 2832, 2035, 1957, 1448, 1305, $1174,1136,1069,928,740,614,590 \mathrm{~cm}^{-1}$.

A solution of the dimethyl ketal obtained above and CAN (1.75 g, $3.2 \mathrm{mmol}, 5.0$ equiv) in anhydrous $\mathrm{MeOH}$ (640 $\mathrm{mL}$ ) was treated at $40{ }^{\circ} \mathrm{C}$ according to the general cycloaddition procedure (method $\mathrm{A}$ ). The resulting crude reaction mixture was purified by $\mathrm{SiO}_{2}$ gel flash column chromatography (10:1 pentanes: $\left.\mathrm{Et}_{2} \mathrm{O}\right)$ to afford cycloadduct 57 as colorless oil (60 mg, 40\%). ${ }^{1} \mathbf{H}$ NMR (400 $\left.\mathrm{MHz}, \mathrm{CDCl}_{3}\right)$ : $\square 6.44(1 \mathrm{H}, \mathrm{d}, J=5.5 \mathrm{~Hz}), 6.00(1 \mathrm{H}, \mathrm{dd}, J=$ $5.5,1.8 \mathrm{~Hz}), 5.68(1 \mathrm{H}$, app pent, $J=1.5 \mathrm{~Hz}), 4.75(1 \mathrm{H}, \mathrm{dd}$, $J=4.8,1.8 \mathrm{~Hz}), 3.30(3 \mathrm{H}, \mathrm{s}), 3.28(3 \mathrm{H}, \mathrm{s}), 2.87(1 \mathrm{H}, \mathrm{dm}$, $J=4.8 \mathrm{~Hz}), 2.12(2 \mathrm{H}, \mathrm{m}), 1.95(1 \mathrm{H}, \mathrm{m}), 1.72(1 \mathrm{H}, \mathrm{m})$, $1.54(3 \mathrm{H}, \mathrm{dd}, J=1.5,1.1 \mathrm{~Hz}) .{ }^{13} \mathbf{C} \mathbf{N M R}(100 \mathrm{MHz}$, $\left.\mathrm{CDCl}_{3}\right): \square 150.0,136.4,133.2,129.0,107.1,94.7,78.7$, $56.0,55.5,51.0,48.6,30.6,25.0,16.1$. IR $(\mathrm{NaCl}$, thin film): 3133, 3033, 2036, 2827, 1681, 1569, 1470, 1299, 1157, 1071, 1035, 883, 765, $597 \mathrm{~cm}^{-1}$. HRMS: $\mathrm{m} / z$ calcd for $\left[\mathrm{C}_{14} \mathrm{H}_{18} \mathrm{O}_{3} \mathrm{Na}\right]^{+}:$257.1154. Found: 257.1163 (ES).

\footnotetext{
${ }^{1}$ (a) Limanto, J.; Snapper, M. L.J. Org. Chem. 1998, 63, 6440. (b) Porter, J. R.; Snapper, M. L. Synthesis 1999, 1407.
} 